\title{
Stick Stippling for Joint 3D Visualization of Diffusion MRI Fiber Orientations and Density
}

\author{
Ryan P. Cabeen ${ }^{1}$, David H. Laidlaw ${ }^{2}$, Arthur W. Toga ${ }^{1}$ \\ ${ }^{1}$ Laboratory of Neuro Imaging (LONI), USC Mark and Mary Stevens Neuroimaging \\ and Informatics Institute (INI), Keck School of Medicine of USC, University of \\ Southern California, Los Angeles, CA USA \\ ${ }^{2}$ Department of Computer Science, Brown University, Providence, RI USA
}

\begin{abstract}
This paper investigates a stick stippling approach for glyphbased visualization of complex neural fiber architecture derived from diffusion magnetic resonance imaging. The presence of subvoxel crossing fibers in the brain has prompted the development of advanced modeling techniques; however, there remains a need for improved visualization techniques to more clearly convey their rich structure. While tractography can illustrate large scale anatomy, visualization of diffusion models can provide a more complete picture of local anatomy without the known limitations of tracking. We identify challenges and evaluate techniques for visualizing multi-fiber models and identified techniques that improve on existing methods. We conducted experiments to compare these representations and evaluated them with in vivo diffusion MR datasets that vary in voxel resolution and anisotropy. We found that stick rendering as 3D tubes increased legibility of fiber orientation and that encoding fiber density by tube radius reduced clutter and reduced dependence on viewing orientation. Furthermore, we identified techniques to reduce the negative perceptual effects of voxel gridding through a jittering and resampling approach to produce a stippling effect. Looking forward, this approach provides a new way to explore diffusion MRI datasets that may aid in the visual analysis of white matter fiber architecture and microstructure. Our software implementation is available in the Quantitative Imaging Toolkit (QIT).
\end{abstract}

Keywords: diffusion MR imaging, neuroimaging, visualization, glyphs, fixels, multi-fiber modeling, fiber orientation distributions

\section{Introduction}

Diffusion magnetic resonance imaging (dMRI) provides a unique probe of water molecule diffusion that can reveal the complex architecture of neural tissue, including crossing, kissing, and bending fibers [1]. This complexity poses a challenge when visualizing dMRI datasets due to the dense and overlapping nature of fiber bundles in the brain [2]. While tractography provides a powerful tool for understanding large scale neural structures, there is high variability across 
tracking methods and false positives are difficult to avoid [3] and there are limitations to its anatomical accuracy [4]. To help provide a more complete picture of the underlying anatomy and to understand why tracking algorithms fail, it can be useful to instead visualize the underlying voxel models representing local diffusion properties in each voxel [5] [6] [7].

Glyph rendering is a common technique for visualizing local features of dMRI data [8]; in this process, markers or symbols are used to visually encode the diffusion or microstructure properties at various positions of a volume. Previous work has included a variety of useful glyphs [2], including ellipsoids [5] and superquadratics representing tensors [9] [10]. Glyphs have also been used to represent more complex models, such as orientation distribution functions (ODFs) [11], and spherical harmonic representations of fiber orientation distributions (FODs) [12] [13]. Other work has focused on directly visualizing fiber organization through glyph packing [14], and two-dimensional techniques such as line rendering [15] [16] and line stippling [17] [18]. Nevertheless, the visualization of complex fiber configurations remains a major challenge, due to cluttering in high resolution data, dependence on viewing orientation, and the introduction of perceptual artifacts due to the gridding of voxels.

Our primary contribution is the development of a visualization technique that combines several of these existing approaches to better depict crossing fiber configurations with fixels, a term we adopt here to refer to the combination of fiber orientations and associated fiber density or volume fraction (Fig 1) [19]. We propose methods that build on related superquadratic and fiber stippling work by using data-modulated 3D stick glyphs and a stippling effect to more clearly depict crossing fiber orientation and density. We compare these and other multifiber glyphs and show how they are affected by image gridding and viewing orientation. We then evaluate this technique by creating visualizations of in vivo neuroimaging data with varying voxel resolution and anisotropy to show the benefits of this approach for typical data exploration sessions.

\section{Methods}

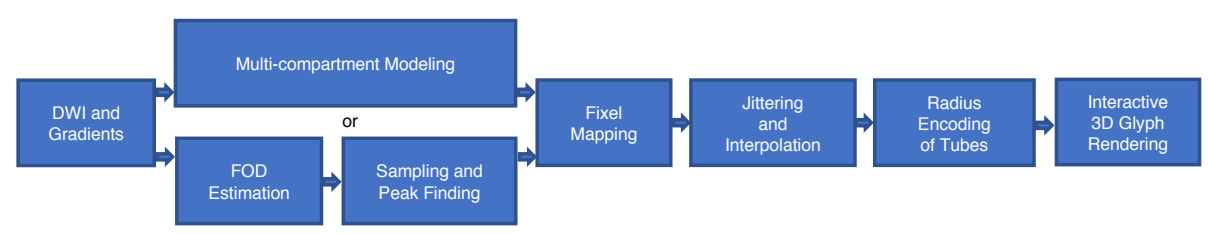

Fig. 1: The proposed computational pipeline for creating stick stippling visualizations from diffusion MRI data. The pipeline applies to both multi-compartment models, such as the ball-and-sticks model, as well as FODs represented by spherical harmonics. 


\subsection{Diffusion Modeling and the Fixel Representation}

We focus here on the visualization of multi-fiber models using a fixel representation that can summarize either FODs [20] or multi-compartment models such as the ball-and-sticks model [21]. Each fixel consists of a fiber orientation and its associated fiber density, which are extracted as follows. For FOD modeling, fiber orientations are extracted by sampling the distribution on a four-fold subdivision of an spherical icosahedral mesh. From this, fiber orientations are extracted from the local maxima on the mesh, and fiber density is taken by the peak density. FOD fixels further processed to remove duplicates at antipodal points and local ridges using hierarchical clustering with a sine angle distance. For multicompartment modeling, fixels are directly derived from the principal direction and volume fraction of each compartment. While previous work has used fixels primarily for FODs [22], we more generally use fixel density to represent either volume fraction or density, depending on the underlying model.

More formally, the fixels of the $i$-th voxel are given by $M_{i}=\left\{\left(f_{i j}, \mathbf{v}_{i j}\right)\right\}_{j=1}^{N_{i}}$, with fixel count $N$, density $f$, and orientation $v$. Our approach also requires interpolation for jittering fixels in a neighborhood around each voxel, and for this, we use a kernel regression framework [23] [24], which estimates the fixels $\hat{M}$ at an arbitrary position $p$ from a neighborhood of voxels $\mathcal{N}$ :

$$
\begin{gathered}
\hat{M}(p)=\underset{M}{\operatorname{argmin}} \sum_{i \in \mathcal{N}} K\left(p_{i}, p\right) d^{2}\left(M_{i}, M\right)+\lambda N \\
d^{2}(M, \hat{M})=\min _{\pi} \sum_{j} f_{j}\left(1-\left(\mathbf{v}_{j} \cdot \hat{\mathbf{v}}_{\pi(\mathbf{j})}\right)^{2}\right) \\
K\left(p_{i}, p\right)=\exp \left(-\left\|p_{i}-p\right\|^{2} / h^{2}\right)
\end{gathered}
$$

given spatial bandwidth $h$, and regularization $\lambda=0.99$. While previous work has used this with ball-and-sticks modeling, we more generally use this here with fixels obtained from FODs [22] as well. The primary goal here is then to develop improved glyph-based visualization techniques based on this approach, which we describe next.

\subsection{Fixel Glyph Visualization}

The design of glyph visualizations generally includes a number of technical challenges related to visual perception [25] [26] [27] [28], and we identified three major issues specifically related to fixels (Fig. 2). First, the complexity of multi-fiber models often creates cluttered scenes, making it difficult to distinguish important fibers from unimportant ones. Second, the viewing orientation of the scene can reduce the legibility of fixel orientation and density, particularly those parallel to the camera's viewing direction. Third, the discretization of the voxel grid can break gestalt principles of perceptual organization, making some bundles appear more organized than others, even when they only differ in orientation relative to the viewing angle. 
bioRxiv preprint doi: https://doi.org/10.1101/2020.06.15.153098; this version posted June 16, 2020. The copyright holder for this preprint (which was not certified by peer review) is the author/funder, who has granted bioRxiv a license to display the preprint in perpetuity. It is made available under aCC-BY-NC-ND 4.0 International license.

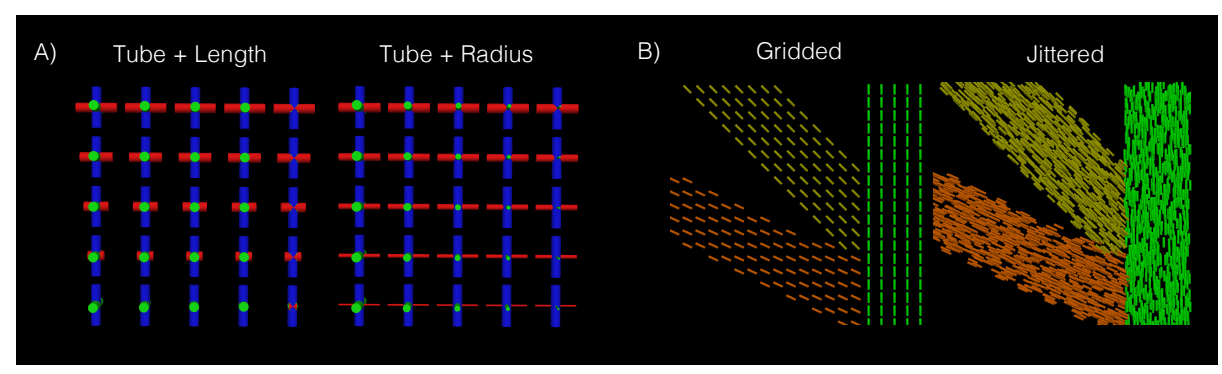

Fig. 2: Perceptual challenges for fixel visualization shown in synthetic data. Panel A shows challenges related to viewing orientation, where length and radius encoding of fiber density are compared. Red fiber density decreases from top to bottom, and green fiber density decreases left to right. This illustrates how the density of fibers parallel to the camera view (green) is only legible with radius encoding. Panel B shows challenges related to voxel discretization, where gridded and jittered glyphs are compared. Three bundles were created with equal thickness but with different orientations. This illustrates how gridding can make axis-aligned bundles look more coherent, while jittering can avoid these issues to give an equally coherent appearance to all bundles.

We propose to address these issues by using the 3D shape and layout of fibers to reduce visual ambiguities (Fig. 1). First, we render fixels as tubes (or sticks) to provide a clearer reading of fiber orientation, as shading from the $3 \mathrm{D}$ shape can provide depth cues, unlike rendering solid lines. We also color fixels according to the widely used RGB-directional color mapping. Second, we modulate the radius of the tube to reflect the fiber density. The advantage of modulating the radius is that it can be appreciated from any viewing orientation, unlike tube length, which can be illegible when parallel to the viewing direction and can be confused with orientation due to perspective foreshortening. Finally, we render fixels using jittering of 3D glyphs to produce a stippling effect, a process that greatly reduces the effect of gridding by drawing glyphs at uniformly sampled points within each voxel. This approach builds on related work on stippling and superquadratics to visualize fixels. Furthermore. In the following section, we conduct experiments to make comparisons with previously used multi-fiber visualizations and to understand how effectively they address the challenges posed in fixel visualization.

\section{$3 \quad$ Experiments and Results}

We conducted experiments with three datasets to understand the strengths and limitations of various fixel rendering techniques with regard to how they represent complex anatomy. These datasets were chosen to include voxels of varied resolution and anisotropy in order to show performance across anatomical scales and imaging parameters. In addition to techniques described in the previous section, we compared the results to solid line rendering, tube length encoding, 
bioRxiv preprint doi: https://doi.org/10.1101/2020.06.15.153098; this version posted June 16, 2020. The copyright holder for this preprint (which was not certified by peer review) is the author/funder, who has granted bioRxiv a license to display the preprint in perpetuity. It is made available under aCC-BY-NC-ND 4.0 International license.

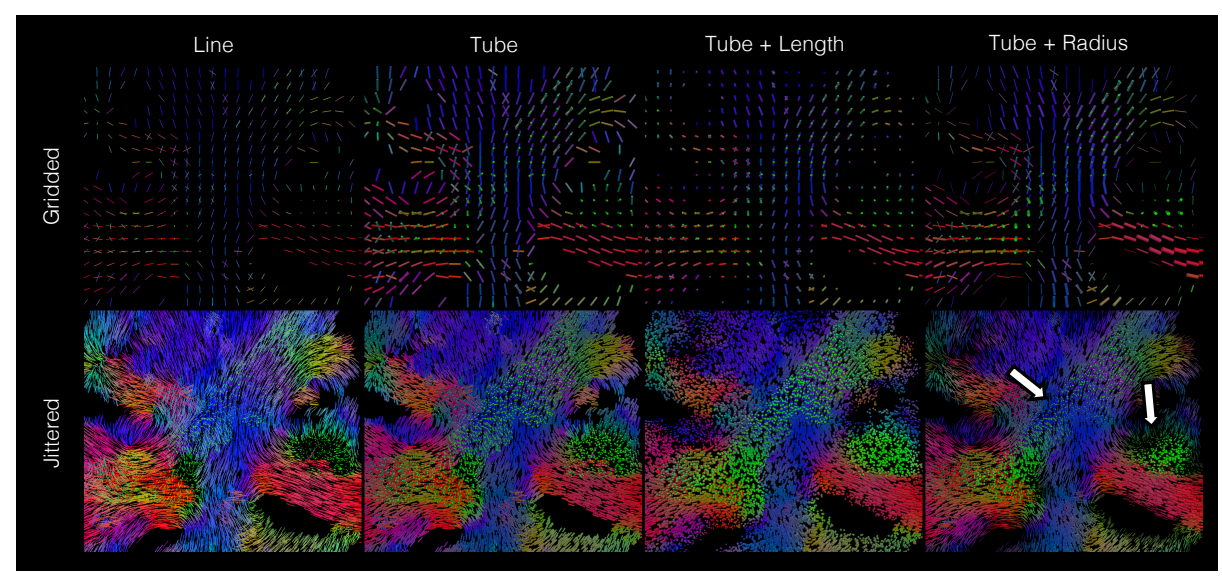

Fig. 3: Eight fixel visualizations with clinically feasible data. A comparison of the first and second columns shows how 3D tube rendering more clearly depicts fiber orientation. A comparison of the third and fourth columns show how radius encoding can illustrate variation in fiber density, e.g. the variation in SLF fibers shown in green (white arrows).

and spherical harmonic FOD rendering when applicable. Our experiments were implemented in the Quantitative Imaging Toolkit (QIT) [29].

\subsection{Clinical Data Experiment}

Our first experiment examined data from a typical clinical acquisition. Diffusionweighted MR image volumes were acquired from a healthy 34 year old volunteer, conducted on a GE $1.5 \mathrm{~T}$ scanner with voxel size of $2 \mathrm{~mm}^{3}$, matrix size $128 \times 128$, and 72 contiguous axial slices. A total of 71 volumes were acquired, with seven $\mathrm{T}_{2}$-weighted volumes (b-value $0 \mathrm{~s} / \mathrm{mm}^{2}$ ) and 64 diffusion-weighted volumes (bvalue $1000 \mathrm{~s} / \mathrm{mm}^{2}$ ) and distinct gradient encoding directions. Ball-and-sticks were estimated using MCMC optimization procedure of Behrens et al. [21].

Eight visualizations were generated to depict a coronal slice showing complex interactions among the corpus callosum (CC), and superior longitudinal fasciculus (SLF), cingulum bundle, and corona radiata (Fig. 3). This figure compares gridded and jittered glyph placement, line and 3D tube drawing, and length and radius encodings of fiber density. We found that jittering effectively removed evidence of the underlying voxel grid, and visualization representing fiber density had less clutter. We found that length encoding of volume fraction was only effective in fibers orthogonal to the viewing direction, however, the density of fibers parallel to the view, such as the SLF and cingulum, were only appreciable with radius encoding. 
bioRxiv preprint doi: https://doi.org/10.1101/2020.06.15.153098; this version posted June 16, 2020. The copyright holder for this preprint (which was not certified by peer review) is the author/funder, who has granted bioRxiv a license to display the preprint in perpetuity. It is made available under aCC-BY-NC-ND 4.0 International license.

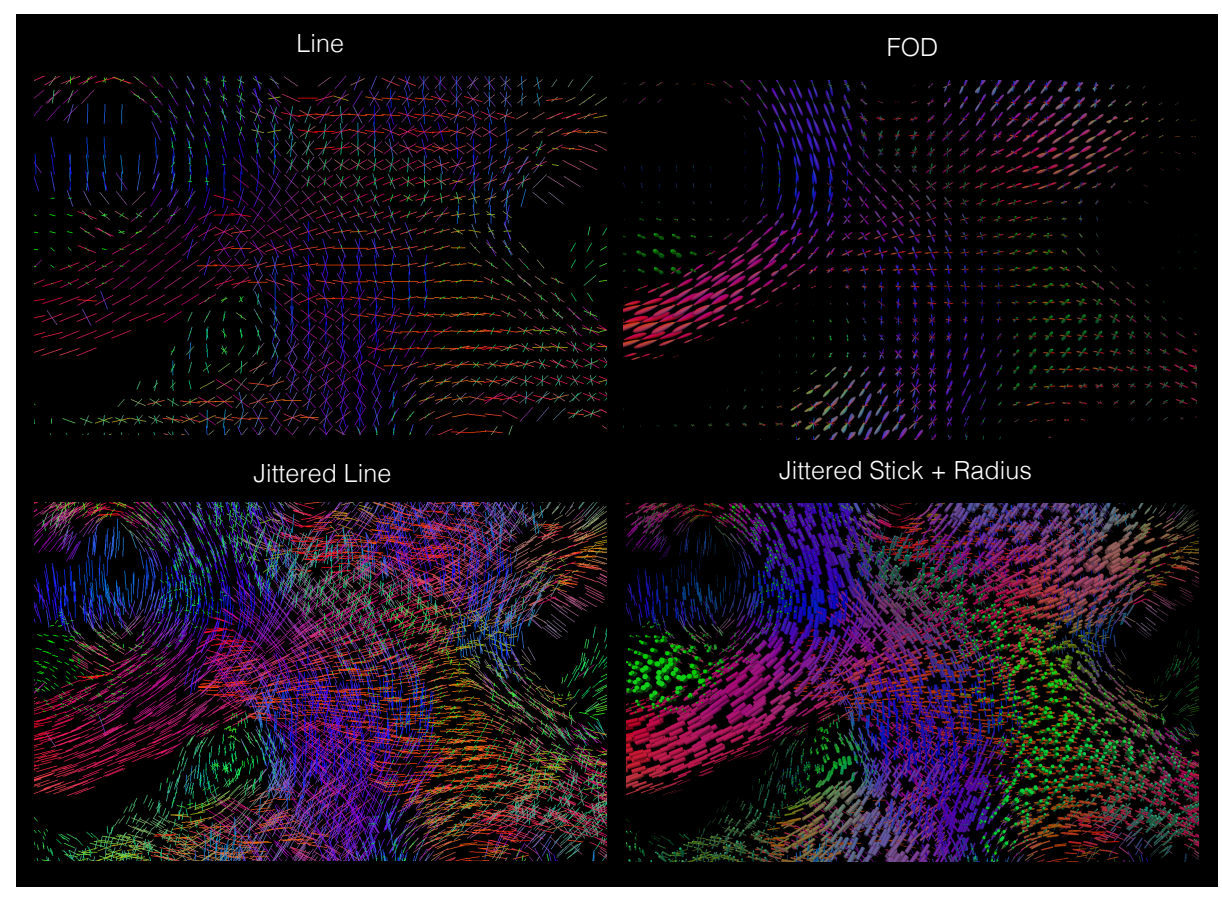

Fig. 4: Visualizations of human connectome project data. The top row shows how high spatial resolution can impact visibility in line and ODF rendering. The bottom right shows how jittering can avoid gridding artifacts and improve visibility.

\section{$3.2 \quad$ HCP Experiment}

Our second experiment examined state-of-the-art dataset from the Human Connectome Project ${ }^{1}$, specifically the single subject dataset with identifier 100307. Diffusion-weighted MR imaging was conducted on a Siemens 3T scanner with voxel size of $1.25 \mathrm{~mm}^{3}$, matrix size $145 \times 145$, and 174 slices. A total of 288 volumes were acquired, with $18 \mathrm{~T}_{2}$-weighted volumes (b-value $0 \mathrm{~s} / \mathrm{mm}^{2}$ ) and the remainder distributed among roughly three shells (b-values 1000, 2000, 3000 $\mathrm{s} / \mathrm{mm}^{2}$ ) with distinct gradient encoding directions. FODs were estimated using the compartmental modeling approach of Tran et al. [30] using 16 order spherical harmonics. The FODs were discretized using a subdivided icosahedron, and fixels were created from the peak directions and fiber density.

Four visualizations were generated to depict a coronal slice that includes a triple crossing of the corticospinal tract, CC, and SLF (Fig. 4). These visualizations included line rendering, spherical FOD rendering, gridding tubes, and jittered tubes. We found that the high resolution of the data make gridded visualizations more difficult to read, due to the smaller size of fixel glyphs. The tube renderings were more legible than either line or FOD rendering, but jittered

\footnotetext{
${ }^{1}$ http://www . humanconnectome.org
} 
bioRxiv preprint doi: https://doi.org/10.1101/2020.06 15.153098; this version posted June 16, 2020. The copyright holder for this preprint (which was not certified by peer review) is the author/funder, who has granted bioRxiv a license to display the preprint in perpetuity. It is made available under aCC-BY-NC-ND 4.0 International license.

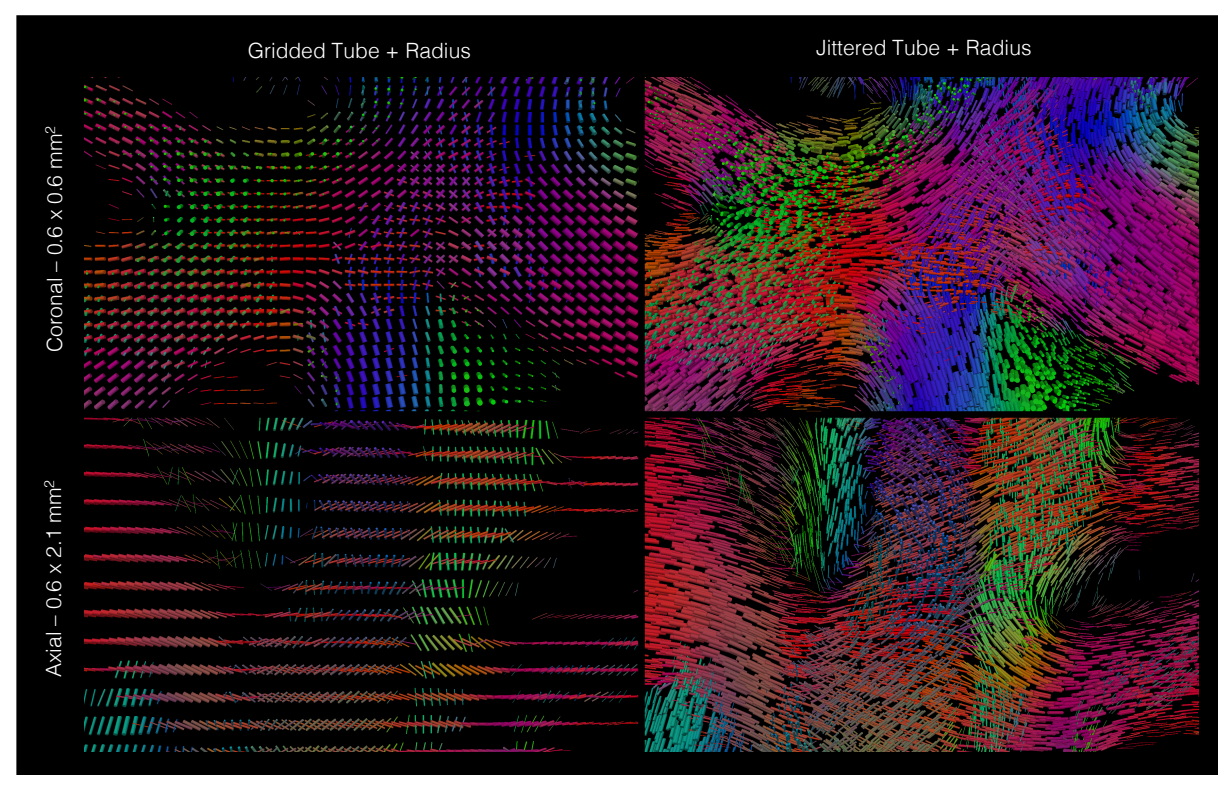

Fig. 5: Visualizations of RESOLVE DWI data. The top left shows how high in-plane resolution can greatly reduce the size and visibility of glyphs. The bottom left shows how this introduces large spacing between glyphs when voxels are anisotropic. By contrast, jittered glyphs were found to greatly reduce these negative effects.

tubes provided the greatest legibility. In particular, the jittering more clearly showed triple crossings, density reductions at the white-gray matter interface and the variation in density of the SLF.

\subsection{RESOLVE Experiment}

Our third experiment examined an advanced presurgical acquisition with highly anisotropic voxels. Diffusion-weighted MR imaging was conducted prior to surgery to treat temporal lobe epilepsy. The RESOLVE DWI sequence was run on a Siemens Prisma 3T scanner with a high resolution coronal in-plane resolution of $0.6 \mathrm{~mm} \times 0.6 \mathrm{~mm}$ with matrix size $272 \times 360.19$ contiguous slices were acquired with thickness $2.1 \mathrm{~mm}$. A total of 56 volumes were acquired, with six $\mathrm{T}_{2}$-weighted volumes (b-value $0 \mathrm{~s} / \mathrm{mm}^{2}$ ) and 50 diffusion-weighted volumes (b-values 800 and $2000 \mathrm{~s} / \mathrm{mm}^{2}$ ) and distinct gradient encoding directions. Ball-and-sticks were estimated using MCMC optimization procedure of Behrens et al. [21] with the continuous exponential model for multi-shell experiments.

Four visualizations were generated to compare fixel visualization of the coronal high in-plane resolution data with an axial slice showing voxel anisotropy (Fig. 5). Each slice was rendered using both gridded and jittered tubes with radius encoding. The coronal slice was found to have fine detail in crossing fibers; however, the axial slice showed perceptual irregularities due to the thick slices. 
Specifically, the anisotropic axial slice showed greater coherence of SLF fibers than CC fibers, due to the greater separation of endpoints of fixels representing the CC. In contrast, the jittered glyphs showed no discrepancy between coronal and axial views, reducing the effects of both voxel gridding and anisotropy.

\section{Discussion and Conclusions}

Our results indicate that when visualizing complex fiber architecture, there are limitations caused by clutter, viewing orientation, and voxel gridding; however, a comparison of glyph visualization techniques showed that these can be mitigated. First, rendering fixels as 3D tube-shaped sticks helped to better indicate orientation through shading and depth cues, and modulating the radius by fiber density helped to reduce clutter and ambiguities based on viewing direction. Second, a stippling effect, produced by glyph jittering and resampling, was found to reduce gridding artifacts, which is perhaps similar to anti-aliasing through randomized glyph placement. Nevertheless, a number of open questions remain. Many applications require the visualization of other per-compartment parameters, e.g. statistical metrics, axon diameter, or myelination, and this requires further work to understand how to effectively encode such parameters with glyphs. There may also be cases where fixels are not a sufficient representation, e.g. when there is fanning or dispersion that may be more apparent by visualizing a complete FOD. There also remain important open problems related to visualizing glyphs in conjunction with tractography [31] and surfaces [32]. Looking forward, the described stick stippling approach may help to guide the design of future imaging studies and aid the development of advanced visualization systems for both human neuroimaging and preclinical imaging [33]. Our software implementation is available online as part of the Quantitative Imaging Toolkit $(\mathrm{QIT})^{23}$.

\section{References}

1. Tuch, D.S., Reese, T.G., Wiegell, M.R., Wedeen, V.J.: Diffusion MRI of complex neural architecture. Neuron 40(5) (2003) 885-895

2. Margulies, D.S., Böttger, J., Watanabe, A., Gorgolewski, K.J.: Visualizing the human connectome. NeuroImage 80 (2013) 445-461

3. Maier-Hein, K., Neher, P., Houde, J.C., Cote, M.A., Garyfallidis, E., Zhong, J., Chamberland, M., Yeh, F.C., Lin, Y.C., Ji, Q., et al.: Tractography-based connectomes are dominated by false-positive connections. bioRxiv (2016) 084137

4. Schilling, K.G., Nath, V., Hansen, C., Parvathaneni, P., Blaber, J., Gao, Y., Neher, P., Aydogan, D.B., Shi, Y., Ocampo-Pineda, M., et al.: Limits to anatomical accuracy of diffusion tractography using modern approaches. NeuroImage 185 (2019) 1-11

\footnotetext{
2 https://cabeen.io/qitwiki

${ }^{3}$ https://resource.loni.usc.edu/resources/downloads/
} 
5. Laidlaw, D.H., Ahrens, E.T., Kremers, D., Avalos, M.J., Jacobs, R.E., Readhead, C.: Visualizing diffusion tensor images of the mouse spinal cord. In: Visualization'98. Proceedings, IEEE (1998) 127-134

6. Zhang, S., Laidlaw, D.H., Kindlmann, G.: Diffusion tensor MRI visualization. The Visualization Handbook (2004) 327-340

7. Tax, C.M., Chamberland, M., van Stralen, M., Viergever, M.A., Whittingstall, K., Fortin, D., Descoteaux, M., Leemans, A.: Seeing more by showing less: Orientationdependent transparency rendering for fiber tractography visualization. PloS one 10(10) (2015) e0139434

8. Vilanova, A., Zhang, S., Kindlmann, G., Laidlaw, D.: An introduction to visualization of diffusion tensor imaging and its applications. In: Visualization and Processing of Tensor Fields. Springer (2006) 121-153

9. Kindlmann, G.: Superquadric tensor glyphs. In: IEEE conference on Visualization, Eurographics Association (2004) 147-154

10. Ennis, D.B., Kindlman, G., Rodriguez, I., Helm, P.A., McVeigh, E.R.: Visualization of tensor fields using superquadric glyphs. Magnetic Resonance in Medicine 53(1) (2005) 169-176

11. Shattuck, D.W., Chiang, M.C., Barysheva, M., McMahon, K.L., De Zubicaray, G.I., Meredith, M., Wright, M.J., Toga, A.W., Thompson, P.M.: Visualization tools for high angular resolution diffusion imaging. In: MICCAI. (2008) 298-305

12. Peeters, T.H., Prckovska, V., van Almsick, M., Vilanova, A., ter Haar Romeny, B.M.: Fast and sleek glyph rendering for interactive hardi data exploration. In: PacificVis 2009, IEEE (2009) 153-160

13. Vaillancourt, O., Chamberland, M., Houde, J.C., Descoteaux, M.: Visualization of diffusion propagator and multiple parameter diffusion signal. In: Visualization and Processing of Higher Order Descriptors for Multi-Valued Data. Springer (2015) 191-212

14. Kindlmann, G., Westin, C.F.: Diffusion tensor visualization with glyph packing. IEEE Trans. on Visualization and Computer Graphics 12(5) (2006)

15. Vaillancourt, O., Boré, A., Girard, G., Descoteaux, M.: A fiber navigator for neurosurgical planning (neuroplanningnavigator). In: IEEE Visualization. Volume 231. (2010)

16. Höller, M., Otto, K.M., Klose, U., Groeschel, S., Ehricke, H.H.: Fiber visualization with LIC maps using multidirectional anisotropic glyph samples. Journal of Biomedical Imaging 2014 (2014) 9

17. Goldau, M., Wiebel, A., Gorbach, N.S., Melzer, C., Hlawitschka, M., Scheuermann, G., Tittgemeyer, M.: Fiber stippling: An illustrative rendering for probabilistic diffusion tractography. In: IEEE BioVis, IEEE (2011) 23-30

18. Goldau, M., Reichenbach, A., Hlawitschka, M.: Visualizing crossing probabilistic tracts. In: IEEE Scientific Visualization, IEEE (2015) 147-148

19. Tournier, J., Calamante, F., Connelly, A., et al.: MRtrix: diffusion tractography in crossing fiber regions. International Journal of Imaging Systems and Technology 22(1) (2012) 53-66

20. Tournier, J.D., Yeh, C.H., Calamante, F., Cho, K.H., Connelly, A., Lin, C.P.: Resolving crossing fibres using constrained spherical deconvolution: validation using diffusion-weighted imaging phantom data. NeuroImage 42(2) (2008) 617-625

21. Behrens, T.E., Berg, H.J., Jbabdi, S., Rushworth, M., Woolrich, M.: Probabilistic diffusion tractography with multiple fibre orientations: What can we gain? NeuroImage 34(1) (2007) 144-155 
22. Raffelt, D.A., Tournier, J.D., Smith, R.E., Vaughan, D.N., Jackson, G., Ridgway, G.R., Connelly, A.: Investigating white matter fibre density and morphology using fixel-based analysis. NeuroImage 144 (2017) 58-73

23. Cabeen, R.P., Bastin, M.E., Laidlaw, D.H.: Kernel regression estimation of fiber orientation mixtures in diffusion MRI. NeuroImage 127 (2016) 158-172

24. Cabeen, R.P., Bastin, M.E., Laidlaw, D.H.: Estimating constrained multi-fiber diffusion $\mathrm{mr}$ volumes by orientation clustering. In: MICCAI. (2013) 82-89

25. Borgo, R., Kehrer, J., Chung, D.H., Maguire, E., Laramee, R.S., Hauser, H., Ward, M., Chen, M.: Glyph-based visualization: Foundations, design guidelines, techniques and applications. In: Eurographics (STARs). (2013) 39-63

26. Wagemans, J., Elder, J.H., Kubovy, M., Palmer, S.E., Peterson, M.A., Singh, M., von der Heydt, R.: A century of gestalt psychology in visual perception: I. perceptual grouping and figure-ground organization. Psychological bulletin 138(6) (2012) 1172

27. Senay, H., Ignatius, E.: Rules and principles of scientific data visualization. Institute for Information Science and Technology, Department of Electrical Engineering and Computer Science, School of Engineering and Applied Science, George Washington University (1990)

28. Fuchs, J., Isenberg, P., Bezerianos, A., Keim, D.: A systematic review of experimental studies on data glyphs. IEEE transactions on visualization and computer graphics (2016)

29. Cabeen, R., Laidlaw, D., Toga, A.: Quantitative Imaging Toolkit: Software for Interactive 3D Visualization, Data Exploration, and Computational Analysis of Neuroimaging Datasets. In: In Proceedings of the International Society for Magnetic Resonance in Medicine (ISMRM). Paris, France. (2018) 2854

30. Tran, G., Shi, Y.: Fiber orientation and compartment parameter estimation from multi-shell diffusion imaging. IEEE TMI 34(11) (2015) 2320-2332

31. Vos, S.B., Viergever, M.A., Leemans, A.: Multi-fiber tractography visualizations for diffusion MRI data. PloS one 8(11) (2013) e81453

32. Goldau, M., Wiebel, A., Hlawitschka, M., Scheuermann, G., Tittgemeyer, M.: Visualizing DTI parameters on boundary surfaces of white matter fiber bundles. In: IASTED. (2011) 53-61

33. Cabeen, R.P., Immonen, R., Harris, N.G., Gröhn, O., Smith, G., Manninen, E., Garner, R., Duncan, D., Pitkänen, A., Toga, A.W.: A computational diffusion mri framework for biomarker discovery in a rodent model of post-traumatic epileptogenesis. In: 2020 IEEE 17th International Symposium on Biomedical Imaging (ISBI), IEEE (2020) 1013-1017 